\title{
PERIODIC ORBITS AND THE CONTINUITY OF ROTATION NUMBERS
}

\author{
RICHARD SWANSON
}

(Communicated by Charles Pugh)

\begin{abstract}
The main result is that an annulus homeomorphism homotopic to the identity either has a well-defined and continuous assignment of rotation numbers on its chain recurrent set or there exists an interval of rotation numbers and periodic points corresponding to each reduced rational number in the interval. As a corollary, rotational discontinuities force the mapping to admit periodic points of all sufficiently large periods $n$. In a related result, we provide a criterion for the rotation set of an annulus homeomorphism to be nowhere dense.
\end{abstract}

For homeomorphisms $f: \mathbb{A} \rightarrow \mathbb{A}$ of the annulus that are homotopic to the identity, the rotation behavior can be predicted from the behavior on individual chain components. A very strong result along these lines, due to Franks [F1], asserts that if a compact chain transitive set $\Lambda$ has points $x$ and $y$ with distinct rotation numbers $\alpha$ and $\beta$, then the given map $f$ has, corresponding to each reduced rational $p / q$ in the interval $(\alpha, \beta)$, a periodic orbit of period $q$. In this paper we will explore the opposite situation of rotational simplicity, which; as we shall shortly see, forces the mapping that assigns a rotation number to each point to be well defined and continuous on the full chain recurrent set $\mathscr{R}(f)$ of the given homeomorphism. In fact, in this note we shall prove the following:

Theorem. Let $f: \mathbb{A} \rightarrow \mathbb{A}$ be a homeomorphism of the annulus, homotopic to the identity map. Let $\mathscr{R}$ denote the chain recurrent set of $f$. Suppose that the set of rotation numbers of periodic orbits of $f$ is nowhere dense. Then the rotation number function $\rho: \mathscr{R} \rightarrow \mathbb{S}^{1}$ is well defined and continuous.

In fact, when the rotation mapping is either ill defined or discontinuous, there are periodic orbits having all periods with the possible exception of some finite set of natural numbers (see the corollary following the proof). The reader should note that when one ranges outside the chain recurrent set, the rotation mapping often fails to be continuous due to the proximity of basins of attraction

Received by the editors April 23, 1991 and, in revised form, May 21, 1991.

1980 Mathematics Subject Classification (1985 Revision). Primary 58J22.

Key words and phrases. Annulus maps, rotation numbers, chain recurrence.

The work was supported in part by a grant from the National Science Foundation. The author would also like to thank the Centre de Recerca Matemàtica at the Universitat Autònoma de Barcelona in Spain. 
of periodic orbits (or invariant circles) with different rotation numbers. I would like to thank Marcy Barge for a helpful discussion concerning the corollary and the referee for a number of good suggestions.

Before passing on to the proof of the theorem, we shall need some ideas and terminology concerning rotation numbers and pseudo-orbits or $\varepsilon$-chains as they are called in [C]. Fix $\varepsilon>0$. An $\varepsilon$-pseudo-orbit is an infinite sequence of points $\left\{z_{k}\right\}$ in $\mathbb{A}$ such that for all $k \geq 0, d\left(z_{k+1}, f\left(z_{k}\right)\right) \leq \varepsilon$. An $\varepsilon$-chain of length $n$ from $x=z_{0}$ to $y=z_{n}$ is a sequence $\left\{z_{k}\right\}$ with $k=0,1,2, \ldots, n$ such that for all $0 \leq k<n$, one has $d\left(z_{k+1}, f\left(z_{k}\right)\right) \leq \varepsilon$.

We shall also need to review the definition of the chain recurrent set of the mapping $f: \mathbb{A} \rightarrow \mathbb{A}$. We say that $x$ is chain equivalent to $y$ if for each $\varepsilon>0$ there exists an $\varepsilon$-chain from $x$ to $y$ and an $\varepsilon$-chain from $y$ to $x$. The point $x$ is called chain recurrent if $x$ is chain equivalent to itself. The chain recurrent set of $f$ is the set of all chain recurrent points, which we shall denote by the symbol $\mathscr{R}$. The relation of chain equivalence is, in fact, an equivalence relation defined on the set $\mathscr{R}[C]$. The resulting equivalence classes are called the chain transitive components of $\mathscr{R}$. A chain transitive set is simply a subset of some chain transitive component.

If $\left\{z_{k}\right\}$ is an $\varepsilon$-chain then we can choose lifts $\tilde{z}_{k}$ of the points $z_{k}$ such that the set of points $\tilde{z}_{k}$ forms an $\varepsilon$-pseudo-orbit for some lift $F: \widetilde{\mathbb{A}} \rightarrow \widetilde{\mathbb{A}}$ of $f$ defined on the covering space $\widetilde{\mathbb{A}}=\mathbb{R} \times[0,1]$. Now define $\rho\left\{z_{k}\right\}$ to be the closed interval of all accumulation points of the sequence $\left\{\pi\left(\tilde{z}_{k}\right) / k\right\}$ where $\pi: \widetilde{\mathbb{A}} \rightarrow \mathbb{R}$ denotes the projection onto the first factor of the covering space. If we want to speak of the "true" rotation set of a point $x \in \mathbb{A}$, we define $\rho(x) \equiv \rho\left\{f^{k}(x)\right\}$. If the set $\rho(x)$ is a single number then we say that $x$ has a rotation number. The rotation number mapping is the real-valued function $x \mapsto \rho(x)$ whose domain will always be some specified subset of points having a rotation number.

Finally, for a compact $f$-invariant set $\Lambda$, define the rotation set of $\Lambda$, $\rho(f, \Lambda)$, consisting of all rotation numbers of points of $\Lambda$. We shall also assume that all rotation numbers have been reduced modulo one, although that is not at all essential in this paper. The proof of the main theorem will require three lemmas.

Lemma 1. Let $R(x)$ denote the chain transitive component of an element $x$ in A. Then $\lim \sup _{y \rightarrow x} R(y) \subset R(x)$. That is, for $\varepsilon>0$ and $y$ sufficiently close to $x$, it follows that $R(y) \subset R(x)$.

Proof omitted. This result holds in any compact metric space, is straightforward, and is essentially in the paper [C].

We remark here that this result is particularly obvious if one uses the equivalent definition, advocated by Franks [F2], of $\varepsilon$-chains in which one allows chains consisting of $\varepsilon / 2$ "jumps" at the beginning and end with a traditional (Conley) $\varepsilon$-chain in between.

Lemma 2. Let $R$ denote a chain transitive component of an annulus homeomorphism $f$ isotopic to the identity. If the rotation set of $\left.f\right|_{R}$ is not a singleton, then the rotation set of $f$ is somewhere dense, i.e., contains an interval.

Proof. This is found in Franks [F1, Corollary 2.4, p. 103]. 
Lemma 3. Suppose that $f: \mathbb{A} \rightarrow \mathbb{A}$ is isotopic to the identity with a lift $F: \widetilde{\mathbb{A}} \rightarrow$ $\widetilde{\mathbb{A}}$. Let $\Lambda$ denote a compact invariant set that lifts to $\widetilde{\Lambda} \subset \widetilde{\mathbb{A}}$. If $\rho\left(\left.F\right|_{\tilde{\Lambda}}\right)$ is contained in $[a, b]$, then for each $\varepsilon>0$ there exists $a \delta>0$ such that each orbit lying in $a \delta$-neighborhood of $\tilde{\Lambda}$ has its rotation set in $[a-\varepsilon, b+\varepsilon]$.

While this result is essentially Lemma 3.4 from [BS], we provide the following self-contained proof suggested by the referee:

Proof. Define $\mathscr{O}(\delta, \tilde{\Lambda})$ to be the set of orbits contained in the $\delta$-neighborhood of $\tilde{\Lambda}$. For $\delta>0$ let $M$ denote the longest string of indices $1 \leq n \leq M$ in any sequence $\left\{p_{n}\right\} \in \mathscr{O}(\delta, \tilde{\Lambda})$ for which $\left(p_{n}-p_{0}\right)_{1} / n \geq b+\varepsilon$. We claim: $F$ is finite. If not, then by compactness of $\Lambda$ and periodicity of the lift, there is an $F$-orbit in $\widetilde{\Lambda}$ such that $\left(F^{n}(p)-p\right)_{1} / n \geq b+\varepsilon$ for all $n \in \mathbb{N}$. Thus $\rho\left\{F^{n}(p)\right\} \geq b+\varepsilon$, a contradiction.

For $n>M$ and an arbitrary orbit $\left\{F^{n}\left(p_{0}\right)\right\} \in \mathscr{O}(\delta, \tilde{\Lambda})$, choose $k_{1}, n-M \leq$ $k_{1} \leq n$, such that $\left(F^{n}\left(p_{0}\right)-F^{k_{1}}\left(p_{0}\right)\right)_{1} /\left(n-k_{1}\right)<b+\varepsilon$. To simplify notation put $p_{n}=F^{n}\left(p_{0}\right)$, for $n \geq 0$.

Repetition results in a sequence $k_{1}>k_{2}>\cdots>k_{r}>0$ such that $k_{t}-k_{t+1} \leq$ $M, k_{r} \leq M$, and $\left(p_{k_{t}}-p_{k_{t+1}}\right)_{1} /\left(k_{t}-k_{t+1}\right)<b+\varepsilon$. Then

$$
\begin{aligned}
\frac{\left(p_{n}-p_{0}\right)_{1}}{n} & \leq \frac{\left(p_{n}-p_{k_{1}}\right)_{1}}{n-k_{1}} \frac{n-k_{1}}{n}+\cdots+\frac{\left(p_{k_{r-1}}-p_{k_{r}}\right)_{1}}{k_{r-1}-k_{r}} \frac{k_{r-1}-k_{r}}{n}+\frac{\left(p_{k_{r}}-p_{0}\right)_{1}}{n} \\
& \leq(b+\varepsilon)\left(\frac{\left(n-k_{1}\right)+\cdots+\left(k_{r-1}-k_{r}\right)}{n}\right)+\frac{\left(p_{k_{r}}-p_{0}\right)_{1}}{n} \leq b+\varepsilon+\frac{K}{n},
\end{aligned}
$$

where the uniform bound $K$ on $\left(p_{k}-p_{0}\right)_{1}, k \leq M$ follows from the uniform continuity of $F$. Thus,

$$
\limsup _{n \rightarrow \infty} \frac{\left(p_{k}-p_{0}\right)_{1}}{n} \leq b+\varepsilon .
$$

Similarly, the lim inf is $\geq a-\varepsilon$.

We are now ready to give the proof of the main theorem stated above.

Proof of the theorem. From Lemma 2 the rotation set of any chain transitive component $R$ in $\mathscr{R}$ is a singleton. This means that the assignment $x \mapsto \rho(x)$ determines a function on $\mathscr{R}$ with values in $\mathbb{S}^{1}$. To prove continuity, fix a point $x \in \mathscr{R}$ and let $y \in \mathscr{R}$ converge to $x$. Choose $\varepsilon$ and $\delta$ as in Lemma 3. By Lemma $1, R(y)$ is eventually in any desired, say $\eta$-, neighborhood of $R(x)$ for $y$ close enough to $x$. But, evidently, true orbits in $R(y)$ are pseudo-orbits that $\delta$-shadow $\left.f\right|_{R(x)}$, for sufficiently small $\eta$. Now by Lemma 3 , their (constant) rotation sets $\rho(x)$ and $\rho(y)$ are within $\varepsilon$.

Corollary. If the rotation number mapping does not define a continuous map on $\mathscr{R}$, then the period set of $f, \operatorname{Per}(f)=\{n: f$ has a point of least period $n\}$ contains all but finitely many numbers.

Proof. We know that there is a point of period $q$ corresponding to each rational number of the form $p / q$ such that $p, q$ are coprime and $\alpha<p / q<\beta$ as in the theorem. We need to establish that the set of numbers $q$ that appears in this fashion consists of all but finitely many natural numbers. This corresponds to the following perfectly number theoretic proposition. 
Proposition 1. Given $0<\alpha<\beta<\infty$, there exists a number $N$ such that for all $n \geq N$, the interval $(\alpha, \beta)$ contains a rational $p / n$ with $p, n$ coprime, i.e., $(p, n)=1$.

Proof. For completeness we supply an elementary proof due to Barge and Gillette (see [BG, Corollary 2.8]). We shall assume all fractions are written in lowest terms. Suppose that $\left[a_{1} / b_{1}, a_{2} / b_{2}\right] \subset(\alpha, \beta)$. Choose $N$ large enough so that if $n \geq N$ and $n=\prod_{i=1}^{\ell} p_{i}^{e_{i}}$ is the prime factorization of $n$ with $p_{1}<p_{2}<\cdots<p_{\ell}$ then either $p_{\ell} \geq 2 b_{1} b_{2}$ or $p_{i}^{e_{i}-1} \geq 2 b_{1} b_{2}$ for some $i$. There are two cases for such $n$.

(1) $n=p_{1}^{e_{1}}$. If $(s, n) \neq 1$ then $(s-1, n)=(s+1, n)=1$. Hence, every closed interval of length $2 / n \leq 1 / b_{1} b_{2}$ contains a fraction $m / n$ with $(m, n)=1$.

(2) $n=\prod_{i=1}^{\ell} p_{i}^{e_{i}}$, with $\ell \geq 2$ : In this case, if $s \prod_{i=1}^{\ell-1} p_{i}^{e_{i}}+1$ is not relatively prime to $n$ then both of the numbers $(s-1) \prod_{i=1}^{\ell-1} p_{i}^{e_{i}}+1$ and $(s+1) \prod_{i=1}^{\ell-1} p_{i}^{e_{i}}+1$ are. Thus, it follows that in every closed interval of length

$$
L=\frac{2 \prod_{i=1}^{\ell-1} p_{i}^{e_{i}}}{n}=\frac{2}{p_{\ell}^{e_{\ell}} \prod_{i=1}^{\ell-1} p_{i}^{e_{i}-1}} \leq \frac{1}{b_{1} b_{2}},
$$

there is a fraction $m / n$ with $(m, n)=1$.

Thus, in every case, for all $n \geq N$ there is a coprime pair $m, n$ with $m / n \in$ $(\alpha, \beta)$.

Let $\sim$ denote the equivalence relation of chain equivalence between two points defined earlier in the paper. Suppose the quotient space $\mathscr{R} / \sim$ is equipped with the quotient topology. Then the projection $\pi: \mathscr{R} \rightarrow \mathscr{R} / \sim$ is continuous. In fact, by Lemma $1, \pi$ is open and $\mathscr{R} / \sim$ is a compact metric space. (For standard details see, for instance, [Du, p. 235].) If $\rho: \mathscr{R} \rightarrow \mathbb{S}^{1}$ is constant on chain transitive components $R$ in $\mathscr{R}$, then $\bar{\rho}: \mathscr{R} / \sim \rightarrow \mathbb{S}^{1}$ is well defined in the commutative diagram

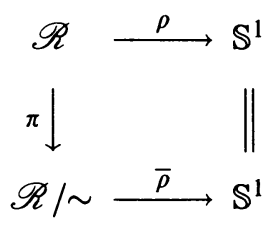

and by the proof of the theorem, $\rho$ and, therefore, $\bar{\rho}$ are continuous. (If they are both well defined, they are continous or discontinuous together.) The existence and properties of complete Lyapunov functions (e.g., [F2]) shows that $\mathscr{R} / \sim$ is compact and totally disconnected. Thus, if $\bar{\rho}$ is continuous and $1-1$, then its homeomorphic image $\bar{\rho}(\mathscr{R} / \sim)=\rho(\mathscr{R})$ must be compact and totally disconnected in $\mathbb{S}^{1}$. Such subsets of the circle are, of course, nowhere dense. We have proven

Proposition 2. Suppose the rotation mapping $\bar{\rho}$ is well defined and 1-1. Then $\rho(\mathscr{R})$ is compact and nowhere dense.

Remark. The reader may find it amusing to construct twist maps such that the rotation map $\bar{\rho}$ is well defined and not $1-1$, and yet the rotation set of $f$ is $\mathbb{S}^{1}$. 


\section{REFERENCES}

[BS] M. Barge and R. Swanson, Rotation shadowing properties of circle and annulus maps, Ergodic Theory Dynamical Systems 8 (1988), 509-521.

[BG] M. Barge and R. Gillette, Rotation and periodicity in plane separating continua, Ergodic Theory Dynamical Systems (to appear).

[C] C. Conley, Isolated invariant sets and the Morse index, CBMS Conference Ser., no. 38, Amer. Math. Soc., Providence, RI, 1978.

[D] J. Dugundji, Topology, Allyn and Bacon, Boston, 1966.

[F1] J. Franks, Recurrence and fixed points of surface homeomorphisms, Ergodic Theory Dynamical Systems 8 (1988), 99-108.

[F2] Periodic points and rotation numbers for area preserving diffeomorphisms of the plane, Inst. Hautes Études Sci. Publ. Math. 71 (1990), 105-120.

Department of Mathematical Sciences, Montana State University, Bozeman, MonTANA 59717 